Journal of Molecular Structure, 84 (1982) 117-128

Elsevier Scientific Publishing Company, Amsterdam - Printed in The Netherlands

\title{
EFFECTS OF ANHARMONICITY OF MOLECULAR VIBRATIONS ON THE DIFFRACTION OF ELECTRONS
}

\author{
Part IV. "Anharmonic shrinkage"
}

\section{LAWRENCE S. BARTELL}

Department of Chemistry, University of Michigan, Ann Arbor, Michigan 48109 (U.S.A.)

(Received 12 February 1982)

\begin{abstract}
Recent observations of anharmonic pseudo-shrinkages in polyatomic molecules greatly exceeding the genuine shrinkage due to vibrational foreshortening have forced a re-examination of anharmonically induced skewing of internuclear distribution peaks for non-bonded distances. It is found that the so called "predictive model" introduced to treat skewing in three-atom fragments $X-A-X$, is entirely inadequate for multiligated cases such as $\mathrm{AX}_{4}$ and $\mathrm{AX}_{6}$. The physical basis of the observed "anharmonic shrinkage" is discussed and a theoretical treatment relating observed peak skewing to anharmonic potential terms is developed. Because practically no spectroscopic or quantum theoretical information exists to test the expressions derived, a simple mechanical model, found in previous studies to have considerable predictive power for quadratic force fields, has been extended to cubic terms. It accounts reasonably well for the observations of anomalous "shrinkages" in hot $\mathrm{CF}_{4}, \mathrm{SiF}_{4}$ and $\mathrm{SF}_{6}$ Accordingly, it appears that diffraction studies may be able to provide new insights into the physical character of molecular force fields.
\end{abstract}

\section{INTRODUCTION}

In recent studies of laser-pumped molecules [1,2] and of thermally excited molecules $[3,4]$, electron diffraction has been shown to provide information that is not easily derived by other methods. If the information latent within diffracted intensities is to be extracted with minimum degradation by systematic errors, however, an attention to rigor in the diffraction analyses is required exceeding that heretofore applied or even feasible in conventional diffraction investigations. One chronic deficiency of diffraction analyses has been a lack of basis for estimating the skewness in probability distributions associated with non-bonded distances. This was forcefully brought to our attention in recent studies [4] of simple, symmetrical molecules at high temperatures. Even after normal "shrinkage" corrections for bond foreshortenings due to perpendicular vibrations had been made, non-bonded distances appeared to be conspicuously shorter than expected from the observed bond lengths. This was puzzling, because we had used the 
non-bonded asymmetry parameters recommended not long ago in a paper [5] that was supposed to provide the first generally applicable answer to the problem in cases where the anharmonic force field is not explicitly known (namely in almost all cases). It turns out that the published answer [5] is totally inadequate in accounting for the effects of anharmonicity beyond those imparted by the stretching anharmonicity and anharmonicity associated with the non-linear transformation between internal coordinates and normal coordinates. Unfortunately, the author had not been sufficiently heedful of the literature where, over a decade ago, the crucial concept involved had not only been recognized and described, but also given the name "anharmonic shrinkage effect [6]." In contrast to the true shrinkage effect of the Bastiansen-Morino variety $[7,8]$, the "anharmonic shrinkage effect" is not a genuine shrinkage effect. It does not shift the center of gravity of non-bonded distribution peaks relative to the bonded peaks by foreshortening. Instead, it skews the peaks and displaces the maxima to distances inside the centers of gravity, making the peak positions appear to shift inward unless an appropriate skew parameter is built into the data refinement. In the original examples $[6,9,10]$ of anharmonic shrinkage the molecules were unusual in several respects and the prominent skewing in their geminal peaks was induced by the coupling of modes of different symmetry brought about by large-amplitude pseudo-rotations. We now see that even in prosaic molecules such as simple tetra- and hexa-halides the effect can be significant, also, and can become plainly evident, at elevated temperatures [4].

It cannot be said that full enlightenment is yet at hand. Such enlightenment will require more information about anharmonicity than is currently known. Nevertheless, it is possible to show in prototype cases at high temperature how the skewing arises, what principal modes are involved, how the peak distortions can be calculated from spectroscopic or quantum theoretic data and how, in the absence of spectroscopic or theoretical data, the effect can be estimated. Such a treatment is outlined in the following sections for simple tetrahedral and octahedral molecules.

\section{QUALITATIVE DESCRIPTION OF EFFECT}

In the original cases $\left(\mathrm{XeF}_{6}, \mathrm{IF}_{7}, \mathrm{ReF}_{7}\right)$ where anharmonic shrinkage effects were observed $[6,9,10]$ it was found that the anharmonic coupling of modes of different symmetry that underlay the effect could be envisaged as "steric" in nature. That is, the signs of the coupling terms were given properly by a picture of atom-atom or bond-bond avoidance. It turns out that the same picture applies to the tetra- and hexa-fluorides studied more recently. Consider, for example, the bending modes belonging to the same irreducible representation as translation in octahedral and tetrahedral molecules, i.e. $t_{1 \mathrm{u}}$ for $\mathrm{O}_{\mathrm{h}}$ and $t_{2}$ for $T_{\mathrm{d}}$ symmetries. While atom pairs swing apart on one side of a molecule, they bump together on the opposite side. Now, if 
molecules vibrated truly harmonically, the closing down of angles would mirror the opening up of angles and the non-bonded atom-atom distributions would be quite symmetric. In actuality, however, atoms offer more resistance to close approaches than to wide separations. Hence, while they may spend approximately half their time in displacements inside the center of gravity, the displacements tend to be smaller than they are on the outside swing where the motion is more unfettered. This constraint on motion skews the distribution to be narrower and therefore higher at negative displacements than it is at positive displacements. In the language of normal coordinates, what has happened is a mixing in, in phase, of modes of different symmetry to relieve the non-Hookean forces encountered at the larger displacements. In an $O_{\mathrm{h}}$ molecule, for example, $t_{2 \mathrm{~g}}$ impurities relieve the backside congestion imparted by $t_{1 \mathrm{u}}$ or $t_{2 \mathrm{u}}$ while, in $T_{\mathrm{d}}, e$ mixing moderates the crowding caused by $t_{2}$. The form of the anharmonic potential function for bond bending associated with this type of relief will be discussed in the following. Naturally, anharmonic components coupling stretches with bends contribute to skewing to some degree but the major contributions beyond those from Morse and non-linear transformation effects appear to be from pure bending.

TREATMENT OF HOT MOLECULES

\section{Approach to distribution function}

For well-characterized force fields the theoretical treatment of anharmonic effects could be carried out as quantitatively as need and resources dictated, along lines developed by Hilderbrandt and $\mathrm{KohI}[11,12]$. Two factors work against such a solution. First, very little information exists about anharmonic potential constants for molecules larger than triatomic. Secondly, even in the simple triatomic cases studied by Hilderbrandt and Kohl, the computer time consumed in deriving and manipulating excited state wavefunctions becomes prodigious at elevated temperatures. Therefore it seems justifiable to introduce a high-temperature approximation which, together with a promising model force field, gives a semiquantitative account of recent observations.

Let the potential function $V$ be expressed as

$$
V=V_{\mathrm{h}}+V_{\mathrm{a}}
$$

where $V_{\mathrm{h}}$ and $V_{\mathrm{a}}$ represent the harmonic and anharmonic components. At high temperatures the probability distribution can be expressed as a Boltzmann distribution in potential energy, or

$$
\begin{aligned}
P_{\mathrm{B}} & =N^{\prime} \exp (-V / k T) \\
& =N^{\prime} \exp \left(-V_{\mathrm{h}} / k T\right) \exp \left(-V_{\mathrm{a}} / k T\right) \\
& =N P_{\mathrm{h}} \exp \left(-V_{\mathrm{a}} / k T\right)
\end{aligned}
$$


where $N^{\prime}$ and $N$ are normalization constants and $P_{\mathrm{b}}$ is the distribution when $V_{\mathrm{a}}$ is neglected.

For the present purposes a further simplification may be made, taking $V_{\mathrm{a}} / \mathrm{kT}$ to be small, and writing

$P_{\mathrm{B}} \approx N P_{\mathrm{b}}\left[1-\left(V_{\mathrm{a}} / k T\right)\right]$

In order to carry out calculations with rigor it is necessary to use rectilinear coordinates as a basis $[13,14]$ among which internal coordinates $R_{i}$, symmetry coordinates $S_{i}$, or normal coordinates $Q_{i}$ are constructed from Cartesian displacement coordinates. Rectilinear coordinates are distinguished from the natural curvilinear stretch and bend coordinates by identifying the latter with circumflexes (e.g. $\bar{R}_{i}$ or $\bar{S}_{i}$ ). The anharmonic potential energy contributions (for which we shall not go beyond cubic terms) are

$$
V_{\mathrm{a}}=\sum_{i} \sum_{j} \sum_{k} f_{i j k} S_{1} S_{k} S_{k} / 6
$$

where each cubic constant can be expressed, as discussed in detail in ref. 14, as

$f_{i j k}=\bar{f}_{i j k}+f_{i j k z}^{\mathbf{T}}$

with $\tilde{f}_{i j k}$ representing the intrinsic anharmonicity in terms of the natural curvilinear stretch and bend coordinates, $\bar{S}_{i}, \bar{S}_{j}$ and $\bar{S}_{k}$, and the second term ansing from the non-linearity of transformations between curvilinear and rectilinear coordinates. As will be explained later, the crucial quantity characterizing the skew of a radial distribution peak is the mean-cube displacement from the mean, or

$$
\begin{aligned}
\left\langle y^{3}\right\rangle & \equiv\left\langle(r-\langle r\rangle)^{3}\right\rangle \\
& =\int y^{3} P_{\mathrm{B}} \mathrm{d} \tau \\
& \approx-N \int y^{3}\left(V_{\mathrm{a}} / k T\right) P_{\mathrm{h}} \mathrm{d} \tau
\end{aligned}
$$

where $d \tau$ is the generalized volume element in displacement coordinates. An even more convenient parameter of skewness for electron diffraction is

$\hat{a} \equiv\left(y^{3}\right) /\left(y^{2}\right)^{2}$

In view of eqns. (4)-(6), it can be seen that this asymmetry parameter $\hat{a}$ can be expressed as a sum of contributions

$$
\begin{aligned}
\hat{a} & =\sum_{i} \sum_{j} \sum_{k} \hat{a}_{i j k} \\
& =\bar{a}+a^{\mathrm{T}}
\end{aligned}
$$

of which we may regard $\bar{a}$ as arising from the cubic anharmonicity in natural internal coordinates and $a^{T}$ as arising from non-linear transformations. In the remainder of this article it will be assumed that the so called "predictive model" of ref. 5 establishes that part of $\hat{a}$ due to Morse anharmonicity $\bar{a}_{M}$ 
and non-linear transformations $a^{T}$, and the emphasis here will be on $\tilde{a}_{\mathrm{b}}$, the remainder of $\hat{a}$ due to bond bending anharmonicity. It may very well be true that a treatment of $\bar{f}_{1 j k}$ (Morse) and $f_{i j k}^{T}$ in the formalism of eqns. (5)-(7) would give a better representation of $\bar{a}_{M}$ and $a^{T}$ than that of ref. 5 . A further improvement extending the treatment to lower temperatures can be made by replacing $P_{\mathrm{h}}$, the Boltzmann distribution in quadratic potential energy, by the distribution

$P_{\mathrm{h}} \propto \Pi_{i} \exp \left(-S_{i}^{2} / 2\left\langle S_{i}^{2}\right\rangle\right)$

where the $\left\langle S_{i}{ }^{2}\right\rangle$ are derived from normal coordinate calculations instead of via the Boltzmann values $\left\langle S_{i}{ }^{2}\right\rangle=f_{i i} / k T$. For the quadratic terms there is no difference between the rectilinear and curvilinear quantities $f_{t i}$ and $\tilde{f}_{i i}$ and no material difference between the $\left\langle S_{i}^{2}\right\rangle$ and $\left\langle\bar{S}_{i}^{2}\right\rangle$.

Shewness and diffraction intensities

First, the relation between the anharmonic potential terms and the intensity of electrons scattered by an anharmonically vibratıng molecule is sought. It is well known [15] that the reduced molecular intensity $M(s)$ is well approximated by a superposition of terms, one for each internuclear distance $r_{i}$, with the form

$$
\begin{aligned}
s M_{i}(s) / G_{i}(s) & =\int_{0}^{\infty}\left[P_{i}(r) / r\right] \sin s r \mathrm{~d} r \\
& =C_{i} \mathrm{e}^{-l \mathrm{~m}^{2} s^{2} / 2}\left[\sin s\left(r_{\mathrm{a}}-\kappa s^{2}-\kappa_{2} s^{4} \ldots\right)\right]_{i} / r_{\mathrm{a}}
\end{aligned}
$$

where $G_{i}(s)$ is a function including scattering factors and phase shifts, $P_{i}(r)$ is the radial distribution function for the internuclear distance in question, $l_{\mathrm{m}}{ }^{2}$ is an effective mean-square internuclear distance nearly equal to $l_{\mathrm{E}}{ }^{2}$ [i.e. the moment $\left.\left\langle(r-\langle r\rangle)^{2}\right\rangle\right]$ and related as explained elsewhere [15], while the constants $\kappa, \kappa_{2}, \ldots$, manifest the anharmonically induced skewing in $P(r)$. In work published to date, there has been no indication that $\kappa_{2}$ and higherorder modulation constants are significant in electron diffraction; therefore, they are neglected. It has become customary to represent the nearly Gaussian peak $P_{i}(r)$ by an expansion of the type

$P(y)=A(\alpha / \pi)^{1 / 2}\left(1+\sum_{n=1}^{\infty} K_{n} y^{n}\right) \exp \left(-\alpha y^{2}\right)$

where, as mentioned in the foregoing, $y$ is defined as the displacement from $r_{\mathrm{g}}$, not $r_{\mathrm{e}}$, and $\alpha$ is taken as $1 / 2 l_{\mathrm{g}}{ }^{2}$ to make $A=1$ up to terms quartic in $l_{\mathrm{g}}$. It is the $K_{n}$ with odd $n$ which skew $P(y)$. In the treatment of ref. 5 it was assumed that the essential skewness was ascribable solely to $K_{3}$ but that is certainly not the case in the present investigation. 
A great simplification, found by inserting eqn. (11) into eqn. (10) and comparing results with those from

$\left\langle y^{3}\right\rangle=\int_{-\infty}^{\infty} y^{3} P(y) d y$

is that

$$
\begin{aligned}
\kappa & =\left\langle y^{3}\right\rangle / 6 A \\
& \approx\left\langle y^{3}\right\rangle / 6
\end{aligned}
$$

with good accuracy, irrespective of how the contributions to $\left(y^{3}\right)$ are distributed among coefficients $K_{3}, K_{5}, K_{7}, \ldots$ Therefore it is convenient in some computations to represent the function $P(y)$ by a specific mathematical form with adjustable mean-square amplitude $l_{\mathrm{g}}{ }^{2}$ and standard coefficient of skewness $A_{3}$, where

$A_{3} \equiv\left(y^{3}\right) / L_{\mathrm{B}}{ }^{3}$

For historical reasons the form of $P(y)$ most commonly adopted has been that of the ground state of a Morse oscillator [16] with potential constant $a$ and mean-square amplitude $l_{\mathrm{g}}$. For the present purposes where an attempt is made to fit a distribution among excited states by such a ground-state function, it is necessary to invoke temperature-dependent parameters $a$ and $l_{\mathrm{g}}$, with the result that $a$ is no longer a (temperature-independent) potential constant. To remind ourselves of this distinction this temperature-dependent parameter is denoted as $\hat{a}$. It should also be carefully noted that the Morse result [17]

$r-r_{\mathrm{e}}=(3 / 2) a l_{\mathrm{g}}^{2}$

valid at any (moderate) temperature for a Morse diatomic oscillator with potential constant $a$, no longer holds for the general oscillator when $a$ is replaced by $\hat{\boldsymbol{c}}$. The analytical expression for the distribution and its eqn. (11)type expansion are given in refs. 15 and 16 . Its standard coefficient of skewness is

$A_{3}=\hat{a} l_{\mathrm{g}}$

and, hence, in terms of $\hat{a}$ and $l_{g}$, the frequency modulation parameter of the diffracted intensity function is very nearly

$$
\begin{aligned}
\kappa & =A_{3} l_{\mathrm{g}}{ }^{3} / 6 \\
& =\hat{a} l_{\mathrm{g}}{ }^{4} / 6
\end{aligned}
$$

the second of which equations was obtained for cold diatomic oscillators a quarter of a century ago [17]. It is fair to ask whether it is realistic to represent an arbitrary skewed distribution by a special Morse function when (1) it is unnecessary and (2) the arbitrary skewed function undoubtedly has an 
appreciably different shape from that of the Morse ground-state distribution. The answers are (1) that several popular computer codes incorporate the Morse function, (2) that it is the coefficient of skewness, $A_{3}$, which matters [cf. eqn. (17a) and the statement under eqn. (13)] and not the exact shape that led to it, and (3) that the Morse function yields the very simple results of eqns. (16) and (17).

Relation between skewness and cubic potential constants

For tetrahedral $\mathrm{AX}_{4}$ molecules with $e$ and $t_{2}$ bending symmetry coordinates $\left(\bar{S}_{2 a}, \bar{S}_{2 b}\right)$ and $\left(\bar{S}_{4 x}, \bar{S}_{4 y}, \bar{S}_{4 z}\right)$, respectively [18], the symmetry-allowed pure-bend cubic constants are $\bar{f}_{222}, \bar{f}_{244}$ and $\tilde{f}_{444}$. Only the first two of these contribute to $\left\langle y^{3}\right\rangle$ and, accordingly, only these are considered. Since all of the six non-bonded distances have precisely the same probability distributions, in the calculation of $\left\langle y^{3}\right\rangle$ it is only necessary to examine one atom pair, say $\mathrm{X}_{1} \cdots \mathrm{X}_{2}$. Through linear terms, the internuclear displacement

$y=q_{12}-(8 / 3)^{1 / 2} R$

where $R$ is $r_{\mathrm{g}}(\mathrm{A}-\mathrm{X})$, can be written for bends as

$y=\left[\left(\tilde{S}_{2 a} / 3\right)+\left(\tilde{S}_{4 z} / 6^{1 / 2}\right)\right] R$

That part of the bending anharmonicity governing moments of the $y$ distribution is, through cubic terms,

$$
\begin{aligned}
V_{\mathrm{a}} & =\frac{1}{6} \tilde{f}_{222}\left(\bar{S}_{2 a}^{3}-3 \tilde{S}_{2 a} \bar{S}_{2 b}^{2}\right) \\
& +\frac{1}{2} \tilde{f}_{244}\left[\bar{S}_{2 a} \bar{S}_{4 z}^{2}-\frac{1}{2} \bar{S}_{2 a}\left(\bar{S}_{4 x}^{2}+\bar{S}_{4 y}^{2}\right)+\cdots\right]
\end{aligned}
$$

In the following the bending contributions to asymmetry parameter $\bar{a}_{\mathrm{b}}$ are derived and it is assumed that the remaining Morse and transformation contributions to $\hat{a}$ and $\kappa$ can be derived by the model of ref. 5 where $\left(\hat{a}_{M}+a^{T}\right)$ is called $a_{\text {eff }}$.

In order to evaluate that part of $\left\langle y^{3}\right\rangle$ (and, hence, of $\hat{a}=\left\langle y^{3}\right\rangle / l_{\mathrm{g}}{ }^{4}$ ) attributable to $f_{222}$ and $f_{244}$, it is sufficient for our purposes to represent $P_{\mathrm{h}}$ of eqn. (3) as

$$
\begin{aligned}
& P_{\mathrm{h}}=P_{\mathrm{I}} \exp \left[-\left(S_{2 a}^{2}+S_{2 b}^{2}\right) /\left(2\left(S_{2}{ }^{2}\right\rangle\right)\right] \times \exp \left[-\left(S_{4 x}^{2}+S_{4 y}^{2}+S_{4 z}^{2}\right) /\right. \\
& \left.\quad\left(2\left\langle S_{4}^{2}\right\rangle\right)\right]
\end{aligned}
$$

where $\left\langle S_{2}{ }^{2}\right\rangle \equiv\left\langle S_{2 a}^{2}\right\rangle=\left\langle S_{2 b}^{2}\right\rangle$ and $\left\langle S_{4}{ }^{2}\right\rangle \equiv\left\langle S_{4 x}{ }^{2}\right\rangle=\left\langle S_{4 y}^{2}\right\rangle=\left\langle S_{4 z}^{2}\right\rangle$ are mean-square values of the symmetry coordinates and $P_{\mathrm{r}}$ is the distribution in all other coordinates. Contributions from $P_{\mathrm{x}}$ cancel in the normalization of $\left\langle y^{3}\right\rangle$. Again, it is not necessary to distinguish between $\left\langle S_{i}{ }^{2}\right\rangle$ and $\left\langle\bar{S}_{i}{ }^{2}\right\rangle$. Insertion of eqns. (19)-(21) into eqn. (6) leads to 
$\bar{a}_{222}=-\left\langle S_{2}{ }^{2}\right\rangle^{3} \bar{f}_{222} R^{3} /\left(36 l_{\mathrm{g}}{ }^{4} k T\right)$

and

$\tilde{a}_{244}=-\left(S_{2}{ }^{2}\right)\left(S_{4}{ }^{2}\right)^{2} \bar{f}_{244} R^{3} /\left(6 l_{\mathrm{g}}{ }^{4} k T\right)$

For octahedral $\mathrm{AX}_{6}$ molecules with $t_{1 \mathrm{y}}, t_{2 \mathrm{~g}}$ and $t_{2 \mathrm{u}}$ symmetry coordinates $\left(\bar{S}_{4 x}, \tilde{S}_{4 y}, \tilde{S}_{4 z}\right),\left(\bar{S}_{5 x}, \bar{S}_{5 y}, \bar{S}_{5 z}\right)$ and $\left(\bar{S}_{6 x}, \bar{S}_{6 y}, \bar{S}_{6 z}\right)$ for bends $[18,19]$, the allowed pure-bend cubic constants are $\bar{f}_{445}, \bar{f}_{456}, \bar{f}_{566}$ and $\bar{f}_{555}$, the latter of which does not contribute to $\left(y^{3}\right)$. For atomic pair $\mathbf{X}_{1} \cdots \mathrm{X}_{2}$, the displacement $y$ is, through linear terms in bends [9]

$y=\left\{8^{1 / 2} \bar{S}_{5 z}+\frac{1}{4}\left[\left(\bar{S}_{4 x}+\bar{S}_{4 y}\right)+\left(\bar{S}_{6 x}+\bar{S}_{6 y}\right)\right]\right\} R$

Bending anharmonicity contributing to $\left\langle y^{3}\right\rangle$ is

$$
\begin{aligned}
V_{\mathrm{a}} & =\bar{f}_{445}\left(\tilde{S}_{4 x} \tilde{S}_{4 y} \bar{S}_{5 z}+\cdots\right)+\tilde{f}_{456} \bar{S}_{5 z}\left(\tilde{S}_{4 y} \bar{S}_{6 x}+\tilde{S}_{4 x} \bar{S}_{6 y}\right) \\
& +\bar{f}_{566}\left(\bar{S}_{5 z} \tilde{S}_{6 x} \tilde{S}_{6 y}+\cdots\right)
\end{aligned}
$$

A treatment exactly analogous to that for tetrahedral molecules yields

$$
\begin{aligned}
& \bar{a}_{445}=C\left(S_{4}{ }^{2}\right)^{2}\left(S_{5}{ }^{2}\right) R^{3} \bar{f}_{445} \\
& \bar{a}_{456}=2 C\left(S_{4}{ }^{2}\right)\left(S_{5}{ }^{2}\right)\left(S_{6}{ }^{2}\right) R^{3} \bar{f}_{456}
\end{aligned}
$$

and

$$
\bar{a}_{566}=C\left\langle S_{5}^{2}\right\rangle\left\langle S_{6}^{2}\right\rangle^{2} R^{3} \bar{f}_{566}
$$

where

$$
C=-18^{1 / 2} /\left(32 l_{\mathrm{g}}{ }^{4} k T\right) \text {. }
$$

Representative numerical examples will be given in a later section.

\section{A model field for estimating cubic constants}

In view of the dearth of published cubic constants, it is necessary to formulate some procedure for estimating them if the equations in the foregoing are $t$, have any utility. One extremely simple model is proposed which has the $v$ tue, at least, of giving the right order of magnitude of the one measured cubir constant available $\left(\bar{f}_{445}\right.$ for $\left.\mathrm{SF}_{6}[20]\right)$ and accounting, as well, quite satisfactc ily for the anharmonic shrinkages of $\mathrm{CF}_{4}$ and $\mathrm{SiF}_{4}$. The model has been strikingly successful in representing quadratic bending constants in cases studied to date $[21,22]$. It is not the purpose of the present article to defend this repelling-points-on-a-sphere (POS) model beyond the above statements, to discuss its application in detail, or to derive the equations presented for force constants below.

It is suggested that the bending potential energy, following the SidgwickPowell-Gillespie-Nyholm "valence shell electron pair repulsion" model [23] can be expressed by a POS formulation 
$V_{\mathrm{POS}}=K \sum_{i} \sum_{j}\left(1 / r_{i j}\right)^{n} \quad K, n>0$

where $r_{i j}$ is the distance between the valence "points-on-a-sphere" and where $K$ and $n$ can be adjusted to make eqn. (30) agree as closely as possible with the known quadratic bending potential surface. Then, for tetrahedral molecules, in units of $(3 / 8)^{n / 2} K / R^{n}$

$f_{22}=n(n+2) / 8$

$f_{44}=n(n+6) / 8$

$\tilde{f}_{222}=-\left(6^{1 / 2} / 192\right) n(n+2)(n+7)$

and

$\tilde{f}_{244}=-\left(6^{1 / 2} / 96\right)\left(n^{3}+9 n^{2}+6 n\right)$

For octahedral molecules, in units of $\left(2 K / R^{n}\right)$, Bernstein and Vernon [24] have shown that

$f_{44}=\left[2^{-n / 2}\left(\frac{n^{2}}{8}+\frac{n}{2}\right)+2^{-n}\left(\frac{n}{8}\right)\right]$

$f_{55}=\left[2^{-n / 2}\left(\frac{n^{2}}{8}+\frac{n}{4}\right)\right]$

and

$f_{66}=\left[2^{-n / 2}\left(\frac{n^{2}}{8}\right)+2^{-n}\left(\frac{n}{8}\right)\right]$

From a table of numerical computations of Bernstein and Vernon, the following expressions accurate to perhaps $1 \%$ have been constructed for cubic constants

$\tilde{f}_{445}=-\left(1.49 \times 10^{-4}\right)\left(n^{4}-21.7 n^{3}-1.3 n^{2}+1890 n-940\right)$

$\bar{f}_{456}=-\left(3.86 \times 10^{-4}\right)\left(n^{3}-64 n^{2}+820 n-1020\right)$

and

$\tilde{f}_{566}=-\left(8.04 \times 10^{-5}\right)\left(n^{4}-12.5 n^{3}-305 n^{2}+4580 n-1430\right)$

in the same energy units as the quadratic constants. A range of $1 \leqslant n \leqslant 11$ applies to eqns. (38) and (40) and $3 \leqslant n \leqslant 9$ to eqn. (39).

\section{Numerical illustrations}

Computations were carried out for $\mathrm{CF}_{4}$ and $\mathrm{SiF}_{4}$ using the force fields of Clark and Rippon [25] and for $\mathrm{SF}_{6}$ with the potential constants of McDowell et al. [26] ; values of $a$, the Morse potential constant, were taken from Herschbach and Laurie [27]. First, values of $K$ and the hardness par- 
ameter $n$ of eqns. (30)-(40) were found which would reproduce the quadratic bending constants of the $\mathrm{AX}_{4}$ molecules exactly and those of $\mathrm{AX}_{6}$ in the mean. Constants $n$ and $a$ are recorded in Table 1 . Next, the force fields were recast into the modified anharmonic Urey-Bradley form $[3,28]$ appropriate for calculation of the Morse and transformation contribution $\left(\bar{a}_{n}\right.$ and $\left.\alpha^{\mathbf{T}}\right)$ via the simplified model of ref. 5 . The resultant values, obtained by Goates [3] are listed in Table 1. Subsequently, cubic constants were evaluated using the POS model of eqns. (33), (34) and (37)-(40). Finally, mean-square amplitudes $\left\langle S_{i}{ }^{2}\right\rangle$ were computed by normal coordinate calculations and inserted into eqns. (22), (23) and (26)-(29) to obtain the values of bending asymmetry parameters $\bar{a}_{b}$ given in Table 1 . For purposes of comparison are listed experimental values of $\hat{a}$ obtained in diffraction analyses. These values were adjusted to make experimental shrinkages fit theoretical shrinkages in recent experiments described elsewhere [4]. Values are preliminary and represent trial refinements of only a small fraction of the total data. It is evident that experimental and model (points-on-a-sphere + Boltzmann + "predictive model") values are in rough agreement with each other. It is not unlikely that a better evaluation of the Morse-non-linearity terms than provided by the "predictive model" of ref. 5 would improve the agreement. A possible procedure for accomplishing this was suggested in the foregoing.

\section{CONCLUSIONS}

The purpose of this paper is to point out that asymmetries in non-bonded radial distribution peaks can be substantial and can cause appreciable errors in deriving non-bonded distances, particularly at elevated temperatures. Compelling arguments, both experimental and theoretical, have been advanced to show that the so called "predictive model" of ref. 5 is entirely

\section{TABLE 1}

Potential constants and non-bonded skew parameters for molecules at various temperatures $\left(\AA^{-1}\right.$ for all $a$ values)

\begin{tabular}{lrllllll}
\hline Molecule & $T(\mathrm{~K})$ & $n^{\mathrm{a}}$ & $a^{\mathrm{b}}$ & $\left(\bar{a}_{M}+a^{\mathrm{T}}\right)^{\mathrm{c}}$ & $\bar{a}_{\mathrm{b}}^{\mathrm{d}}$ & $\hat{a}_{\text {tot }}{ }^{\mathrm{e}}$ & $\hat{a}_{\mathrm{tot}}(\operatorname{expt.})^{\mathrm{f}}$ \\
\hline $\mathrm{CF}_{4}$ & 1600 & 6.5 & 2.0 & 0.0 & 2.6 & 2.6 & 3.0 \\
$\mathrm{SiF}_{4}$ & 900 & 3.8 & 1.9 & -1.0 & 1.6 & 0.6 & 0.9 \\
$\mathrm{SF}_{6} \mathrm{E}$ & 780 & 6.3 & 1.6 & 0.0 & 1.9 & 1.9 & 3.5 \\
& 1700 & 6.3 & 1.6 & 0.1 & 1.8 & 1.9 & 3.7 \\
\hline
\end{tabular}

${ }^{2}$ Hardness exponent, points-on-a-sphere potential function, eqn. (30). bMorse potential constant. "Morse and non-linear transformation contributions to skew parameter, eqn. (8), calculated according to the crude model of ref. 5 . 'Bending anharmonicity contributions to skew according to points-on-a-sphere eqns. (22), (23) and (26)-(28). ${ }^{e}$ Sum of $\left(\bar{a}_{M}+a^{T}\right)$ and $\bar{a}_{b}$. Freliminary electron diffraction results. ${ }^{\mathbf{F}}$ Results pertain to $F-F$ (cis). 
inadequate to represent the total effect of skewing in non-bonded distances in molecules langer than triatomic. Too little spectroscopic information is available to allow precise computations to be made, yet, but a promising, simple model has been proposed which leads to useful estimations of magnitudes.

The approximation introduced to make computations tractable in this preliminary exploration consists of taking a Boltzmann distribution in potential energy truncated to $\left(1-V_{\mathrm{a}} / k T\right)$ in the anharmonic factor. This must fail, of course, at low temperatures when the distribution is largely dictated by zero-point vibrations and at extremely high temperatures where $V_{\mathrm{a}}$ is proportional to the cube of displacements and $k T$ is proportional to only the square. But over a large and reasonable range the approximation should be satisfactory. In this range the convenient skew parameter $\bar{a}_{b}$ for bending is roughly independent of temperature as can be seen in eqns. (22), (23) and (26)-(28) where each mean-square amplitude is approximately proportional to $k T$. Although parameters $\bar{a}_{\mathrm{b}}$ and $\hat{a}$ may not be strong functions of temperature, their influence on derived mean bond lengths increases rapidly with temperature because the intensity modulation parameter $\kappa$ of eqn. (17) is proportional, approximately, to â times $T^{2}$.

Errors introduced into analyses of internuclear distances in $\mathrm{CF}_{4}, \mathrm{SiF}_{4}$ and $\mathrm{SF}_{6}$ when $\hat{a}$ was neglected or when only the $\hat{a}$ of ref. 5 was included were approximately proportional to $T$ and of the order of $0.01 \AA$ at $1500 \mathrm{~K}$. Because such an error may be enormously greater than that due to random intensity errors, it would seem fitting to develop a procedure to compensate for it satisfactorily.

\section{ACKNOWLEDGEMENT}

This research was supported by the National Science Foundation under grant number CHE-7926480. I thank L. S. Bernstein for the use of some of his unpublished points-on-a-sphere potential constants.

\section{REFERENCES}

1 L. S. Bartell, M. A. Kacner and S. R Goates, J. Chem. Phys., 75 (1981) 2730; J. Chem. Phys., 75 (1981) 2736.

2 M. M. Arvedson and D. A. Kohl, Chem. Phys. Lett., 64 (1979) 119; J. Chem Phys., submitted

3 S. R. Goates and L. S. Bartell, J. Chem. Phys., in press.

4 S. R. Goates and L. S. Bartell, J Chem. Phys., in press.

5 L. S. Bartell, J. Mol. Struct., 63 (1980) 259.

6 E. J. Jaco b and L. S. Bartell, J. Chem. Phys., 53 (1970) 2235.

7 Y. Morino, Acta Crystallogr., 13 (1960) 1107

8 S. J. Cyvin, Molecular Vibrations and Mean Square Amplitudes, Elsevier, Amsterdam, 1968.

9 L. S. Bartell and R. M. Gavin, Jr., J. Chem. Phys., 48 (1968) 2466.

10 W. J. Adams H. B. Thompson and L. S. Bartell, J. Chem Phys., 53 (1970) 4040. 
11 R. L. Hilderbrandt and D. A. Kohl, J. Mol. Struct., Theochem, 85 (1981) 25.

12 D. A. Kohl and R. L. Hilderbrandt, J. Mol. Struct., Theochem, 85 (1981) 325.

13 For the necessity of Cartesian displacement coordinates, see L. S. Bartell, J. Chem. Phys., 38 (1963) 1827.

14 L. S. Bartell and S. Fitzwater, J. Chem. Phys., 57 (1977) 4168.

15 K. Kuchitsu and L. S. Bartell, J. Chem. Phys., 35 (1961) 1945.

16 P. M. Morse, Phys. Rev., 34 (1929) 57.

17 L. S. Bartell, J. Chem. Phys., 23 (1955) 1219.

18 To avoid any confusion that might arise concerning the radius of the sphere in the points-on-a-sphere model, introduced to treat anharmonicity, and its relation to the bond length, symmetry coordinates expressed in radians, not distance are adopted.

19 Symmetry coordinates are those illustrated in Fig. 3 of ref. 9, numbering scheme of Fig. 1, except that $a, b, c$ have become $z, x, y$, and the signs of $t_{2 E}$ coordinates and $S_{6 c}$ have been changed.

20 R. K. Heenan, Ph.D. thesis, University of Reading, Reading, Gt. Britain, 1979.

21 L. S. Bartell and V. Plato, J. Am. Chem. Soc., 95 (1973) 3097; L. S. Bartell, Kem. Kozl., 43 (1975) 497.

22 L. S. Bartell, M. J. Rothman and A. Gavezzotti, J. Chem. Phys., 76 (1982) 4136.

23 R. J. Gillespie, Molecular Geometry, Van Nostrand-Reinhold, New York, 1972.

24 L. S. Bernstein and M. Vernon, unpublished work; M. Vernon, J. Mol. Spectrosc., 74 (1979) 102.

25 R. J. H. Clark and D. M. Rippon, J. Mol. Spectrosc., 44 (1972) 479.

26 R. S. McDowell, J. P. Aldridge and R. F. Holland, J. Phys. Chem., 80 (1976) 1203.

27 D. R. Herschbach and V. W. Laurie, J. Chem. Phys., 35 (1961) 458.

28 K. Kuchitsu and L. S. Bartel, J. Chem. Phys., 36 (1962) 2460; J. Chem. Phys., 36 (1962) 2470. 